\title{
Iterative Tomographic Solution of Integer Least Squares Problems with Applications to MIMO Detection
}

\author{
.Jacob Goldberger and Amir Leshem senior member
}

\begin{abstract}
Least squares (LS) fitting is one of the most fundamental techniques in science and engineering. It is used to estimate parameters from multiple noisy observations. In many problems the parameters are known a-priori to be bounded integer valued, or they come from a finite set of values on an arbitrary finite lattice. Integer least squares is also an important problem in multichannel communication systems and GPS applications. In this case finding the closest vector becomes NP-hard problem. In this paper we propose two novel algorithms, the Tomographic Least Squares Decoder (TLSD), that not only solves the ILS problem, better than other sub-optimal techniques, but can also provide the a-posteriori probability distribution for each element in the solution vector and a belief propagation version termed two-dimensional belief propagation (2DBP). Both algorithms are based on reconstruction of the vector from multiple twodimensional projections. The projections are carefully chosen to provide low computational complexity. We show that the projections are equivalent to the two-dimensional marginals of the soft zero forcing linear decoder. We also provide simulated experiments comparing the algorithm to other sub-optimal algorithms. We end with a discussion of possible extensions to coded systems and combinations with sphere decoding.
\end{abstract}

Index Terms-Integer Least Squares, Bayesian decoding, sparse linear equations, subset sum problems, MIMO communication systems, MIMO-OFDM systems, approximate maximum likelihood.

\section{INTRODUCTION}

The problem of finding least squares fit to data is a well known problem, with applications in almost every field of science. When there are no restrictions on the variables the problem has a closed form solution [1]. In many cases, apriori knowledge of the value of the variables is known. One example is the existence of priors, which leads to Bayesian estimators. Another example that is of great interest in many applications is when the variables are either constrained to be integers or constrained to a discrete finite set. This problem has many diverse applications such as decoding of multi-inputmulti-output (MIMO) digital communication systems, GPS system ambiguity resolution [2], radar interferometry [3] and many lattice problems in computer science, such as finding the closest vector in a lattice to a given point in $R^{n}$ [4], and vector subset sum problems which have applications to cryptography

The material in this work was presented in part at the ICASSP conference, Taipei, Taiwan, April 2009.

J. Goldberger is with the School of Engineering, Bar-Ilan University, 52900, Ramat-Gan. Email: goldbej@eng.biu.ac.il

A. Leshem is with the School of Engineering, Bar-Ilan University, 52900, Ramat-Gan. Email: leshema@eng.biu.ac.il
[5]. In contrast to the unconstrained least squares problem, this problem is known to be NP hard [6]. Since the problem is computationally hard, many suboptimal solutions have been proposed over the years. These solutions, range from simple linear inversion known as Zero-Forcing (ZF), combinations of linear reduction and sequential decoding [7], to clever search techniques over the tree of possible solutions, known as sphere decoding [8], [9]. The earlier solutions suffer from significant loss due to noise amplification and work poorly on ill-conditioned matrices, while the latter have exponential average complexity [10]. A good overview of sphere decoding and lattice reduction techniques can be found in [4]. The sphere decoder has also reduced complexity versions such as the partial marginalization of Larsson and Jalden [11]. Another important family is the semidefinite relaxation based decoders [12] [13] [14]. These decoders are based on a simple relaxation of the likelihood function, where the quadratic function is linearized under a rank-one constraint, which is then relaxed. The complexity of the semi-definite decoders is polynomial; however the degree is relatively high, and the solution is quite complex. Low-complexity detectors based tree search and list detection have been proposed in [15] [16] [17] [18] [19] [20].

In this paper we propose an iterative technique, which we dub the Tomographic Least Squares Decoder (TLSD), and the Two-dimensional Belief Propagation (2DBP) which can be used to provide good suboptimal solutions to the general integer least squares problem. To that end, we divide the problem into a large set of partially overlapping sub-problems using projections on many two dimensional subspaces, where each subproblem can be solved optimally. We use all projections onto low-dimensional subspaces that eliminate interference from all but two columns of the channel matrix. Each of the solvers of the sub-problems provides information to the other solvers. The process is iterated until we converge to an a-posteriori probability distribution for each of the variables. The solution is then obtained by assigning to each of the variables the symbol that maximizes its aposteriori probability. The solution also allows us to provide aposteriori probability distributions to each bit of each variable, something desirable in coded communication systems. Such probabilities are hard to evaluate using sphere decoding types of solutions. The major advantage of our approach over that of [21] is that we alleviate the requirement for a banded channel matrix. Furthermore, in contrast to loopy belief propagation, our algorithm has ensured convergence, since we show that it can be interpreted as an instance of the EM Algorithm 
operating on a specific likelihood function. We demonstrate the performance of the proposed method through simulations, where we compare it to several sub-optimal solutions as well as the maximum likelihood solution (when the alphabet size is 2 or 4). A preliminary version of this paper appeared in [22].

The paper proceeds as follows. In Section 2 we provide a formal definition of the integer least squares problem and review previous algorithms. In Section 3 we discuss graphical model approaches that solve the ILS problem. In Section 4 we present the two-dimensional projections approximation and apply the BP algorithm to the approximated model. In Section 5 we present the TLSD decoder as a special case of the EM algorithm and therefore show that it always converges. Experimental results are shown and discussed in Section 6.

\section{PROBlem Formulation}

We consider a MIMO communication system with $n$ transmit antennas and $m$ receive antennas. Denote the MIMO channel matrix by $\mathbf{H}$. The $i j$ entry of $\mathbf{H}$ is the tap gain from transmit antenna $j$ to receive antenna $i$. In each use of the MIMO channel a vector $x=\left(x_{1}, \ldots, x_{n}\right)^{\top}$ is independently selected from a finite set of complex numbers $\mathcal{A}$ according to the data to be transmitted, so that $x \in \mathcal{A}^{n}$. We further assume that in each use of the MIMO channel, $x$ is uniformly sampled from $\mathcal{A}^{n}$. The received vector $y$ is given by

$$
y=\mathbf{H} x+\epsilon .
$$

Here, noise is modeled by the random vector $\epsilon$ which is independent of $x$ and whose components are assumed to be i.i.d. according to a complex Gaussian distribution with zero mean and a known variance $\sigma^{2}$. The $m \times n$ matrix $\mathbf{H}$ comprises i.i.d. elements drawn from a complex normal distribution of unit variance. The MIMO detection problem consists of finding the unknown transmitted vector $x$ given $\mathbf{H}$ and $y$. The task, therefore, boils down to solving a linear system in which the unknowns are constrained to a discrete finite set. The well known maximum-likelihood (ML) estimator in this case is given by

$$
\hat{x}=\arg \min _{x \in \mathcal{A}^{n}}\|y-\mathbf{H} x\|^{2} .
$$

Although the maximum likelihood estimator has very good performance, its complexity grows exponentially with $n$. Similarly, when the noise covariance is a general known matrix $\Sigma$ we can whiten the noise and the ML estimator becomes:

$$
\hat{x}=\arg \min _{x \in \mathcal{A}^{n}}(y-\mathbf{H} x)^{\top} \Sigma^{-1}(y-\mathbf{H} x) .
$$

Pre-multiplying the measurement vector by $\mathbf{R}^{-1}$ where $\Sigma=$ $\mathbf{R R}^{\top}$ is the Choleski decomposition of $\mathbf{C}$, yields problem (2) again. Note that in the case where the linear system is complex-valued we can use a standard method to translate it into an equivalent double-size real-valued representation that is obtained by considering the real and imaginary parts separately:

$$
\left[\begin{array}{l}
\operatorname{Re}(y) \\
\operatorname{Im}(y)
\end{array}\right]=\left[\begin{array}{rr}
\operatorname{Re}(\mathbf{H}) & -\operatorname{Im}(\mathbf{H}) \\
\operatorname{Im}(\mathbf{H}) & \operatorname{Re}(\mathbf{H})
\end{array}\right]\left[\begin{array}{l}
\operatorname{Re}(x) \\
\operatorname{Im}(x)
\end{array}\right]+\left[\begin{array}{l}
\operatorname{Re}(\epsilon) \\
\operatorname{Im}(\epsilon)
\end{array}\right] .
$$

Hence we assume hereafter that $\mathbf{H}$ has real values without any loss of generality.

A second related problem is the Bayesian version, where given an a-priori distribution on the components of the solution, we would like to find the a-posteriori distribution of the vectors, given the measurement vector $y$. This problem appears naturally in the decoding of coded multiple antenna systems, where a first step channel decoding amounts to computing aposteriori probabilities and the log-likelihood ratio for each transmitted bit.

Problem (2) can also be viewed as the closest vector problem [4]; denote the columns of the matrix $\mathbf{H}$ by $\mathbf{h}_{1}, \ldots, \mathbf{h}_{n}$, and find $x_{1}, \ldots, x_{n} \in \mathcal{A}$ such that $\sum_{i=1}^{n} \mathbf{h}_{i} x_{i}$ is closest to the vector $y$.

The optimal maximum-likelihood decoding has exponential computational complexity which makes it unfeasible when either the number of variables or the finite alphabet size are large. In fact, for a general $\mathbf{H}$, the problem is known to be exponentially complex both in the worst-case sense [23] as well as in the average sense [24]. There are many suboptimal solutions to the problem [4]. Many of these are based on sphere decoding by pruning the tree of possible coefficients. This solution has complexity that is exponential in $n$ [10] and therefore hard to implement for large problems, especially, if the alphabet size is large, (as is typical MIMO systems where constellation size of 64 is common). Moreover, these solutions while providing the closest point do not easily provide likelihood ratios per symbol or per bit a-posteriori probabilities. Reduced complexity solutions include among other semidefinite relaxation [12][13] and lattice reduction based techniques [25]. Since these algorithms find the closest point in the lattice by relaxed optimization, it is harder to compute a-posteriori probabilities per symbol or per bit, which is required, when forward error correction is used (e.g., in communication applications), however see [26] for a soft decoding using sphere decoder.

A simple sub-optimal solution, known as the zero-forcing algorithm, is based on a linear decision that ignores the finiteset constraint

$$
z=\left(\mathbf{H}^{\top} \mathbf{H}\right)^{-1} \mathbf{H}^{\top} y
$$

and then, neglecting the correlation between the symbols, finds the closest point in $\mathcal{A}$ for each symbol independently

$$
\hat{x}_{i}=\arg \min _{a \in \mathcal{A}}\left|z_{i}-a\right| .
$$

This scheme performs poorly due to its inability to handle ill-conditioned realizations of the matrix $\mathbf{H}$ (if $m<n$ then $\mathbf{H}^{\top} \mathbf{H}$ is even singular). Somewhat better performance can be obtained by using a minimum mean square error (MMSE) Bayesian estimation for the continuous linear system. Let $e$ be the mean symbol energy. We can partially incorporate the information that $x \in \mathcal{A}^{n}$ by using the prior Gaussian distribution $x \sim \mathcal{N}(0, e I)$. The MMSE estimation becomes

$$
E(x \mid y)=\left(\mathbf{H}^{\top} \mathbf{H}+\frac{\sigma^{2}}{e} I\right)^{-1} \mathbf{H}^{\top} y
$$

and then the finite-set solution is obtained by finding the closest lattice point in each component independently. A 
vast improvement over the linear approaches described above can be achieved by the MMSE with Successive Interference Cancelation (MMSE-SIC) algorithm that is based on sequential decoding with optimal ordering [27]. These linear type algorithms can also easily provide probabilistic (soft-decision) estimates for each symbol. However, there is still a significant gap between the detection performance of the MMSE-SIC algorithm and the performance of the ML detector.

Many alternative methods have been proposed to approach ML detection performance. The sphere decoding (SD) algorithm finds the exact ML solution by searching for the nearest lattice point [28], [29], [30], [31]. Although SD reduces computational complexity compared to the exhaustive search of the ML solution, sphere decoding is not feasible for high-order QAM constellations. While SD has been empirically found to be computationally very fast for small to moderate problem sizes (say, for $n<20$ for 16-QAM), the sphere decoding complexity would be prohibitive for large $n$, higher order QAM and/or low SNRs [32]. Thus, there is still a need for low complexity detection algorithms that can achieve near optimal performance and can compute a-posteriori probabilities per symbol or per bit.

The projection receiver in [33] [34] [35][36] is related to the orthogonal projections approach suggested in our work. The geometrical interpretation of the zero-forcing detector has also been specifically addressed in [37]. Finally the Probabilistic Data Association (PDA) [38],[39] is an iterative algorithm, based on the one-dimensional marginal distributions of the various variables. The approach in this paper is related to the PDA since it also uses marginalization of the variables. However we propose to use two-dimensional marginal distributions. This better captures the relationship between the variables and provides much better performance. Latsoudas and Sidiropoulos [40] proposed combining the PDA with sphere decoding, by using the a-posteriori probabilities provided by the PDA to initialize a sphere decoder. The same technique applies in our case as discussed in the concluding remarks.

\section{The Loopy Belief Propagation ApproACH}

Given the constrained linear system $y=\mathbf{H} x+\epsilon$, and a uniform prior distribution on $x$ over a finite set of points $\mathcal{A}$, the posterior probability function of the discrete random vector $x$ given $y$ is

$$
p(x \mid y) \propto \exp \left(-\frac{1}{2 \sigma^{2}}\|\mathbf{H} x-y\|^{2}\right), \quad x \in \mathcal{A}^{n} .
$$

The notation $\propto$ stands for equality up to a normalization constant. Observing that $\|\mathbf{H} x-y\|^{2}$ is a quadratic expression, it can be easily verified that $p(x \mid y)$ can be factorized into a product of two- and single-variable potentials

$$
p\left(x_{1}, . ., x_{n} \mid y\right) \propto \prod_{i} \psi_{i}\left(x_{i}\right) \prod_{i<j} \psi_{i j}\left(x_{i}, x_{j}\right)
$$

such that

$$
\begin{gathered}
\psi_{i}\left(x_{i}\right)=\exp \left(-\frac{1}{2 \sigma^{2}} y^{\top} \mathbf{h}_{i} x_{i}\right) \\
\psi_{i j}\left(x_{i}, x_{j}\right)=\exp \left(-\frac{1}{\sigma^{2}} \mathbf{h}_{i}^{\top} \mathbf{h}_{j} x_{i} x_{j}\right)
\end{gathered}
$$

where $\mathbf{h}_{i}$ is the $i$-th column of the matrix $\mathbf{H}$. Since the obtained factors are simply a function of pairs of random variables, we obtain a Markov Random Field (MRF) representation [41]. In the MIMO application the (known) matrix $\mathbf{H}$ is randomly selected and therefore, the MRF graph is usually a completely connected graph. (see an MRF graph illustration in Fig. 1).

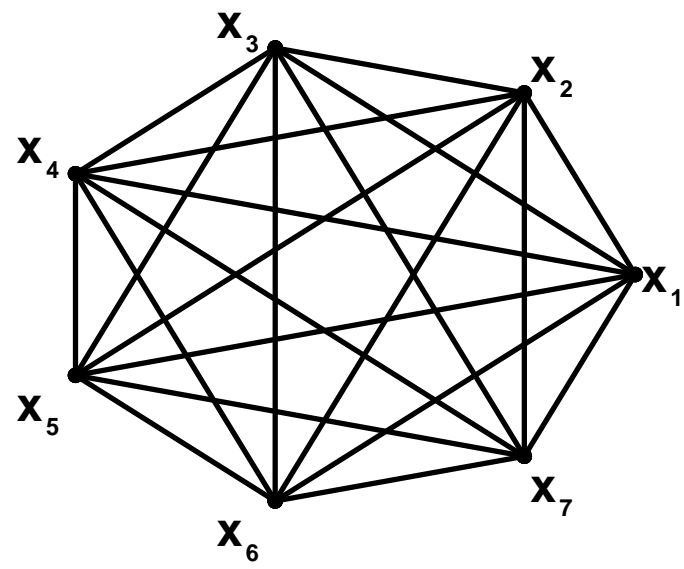

Fig. 1. The MRF undirected graphical model corresponds to the MIMO detection problem with $n=7$.

The Belief Propagation (BP) algorithm aims to solve inference problems by propagating information throughout this MRF via a series of messages sent between neighboring nodes (see [42] for an excellent tutorial on BP). In the sum-product variant of the BP algorithm applied to the MRF (9), the message from $x_{j}$ to $x_{i}$ is

$m_{j \rightarrow i}\left(x_{i}\right)=\sum_{x_{j} \in \mathcal{A}}\left(\psi_{j}\left(x_{j}\right) \psi_{i j}\left(x_{i}, x_{j}\right) \prod_{k \neq i, j} m_{k \rightarrow j}\left(x_{j}\right)\right), \quad x_{i} \in \mathcal{A}$.

In each iteration messages are passed along all the graph edges in both edge directions. In every iteration, an estimate of the posterior marginal distribution ('belief') for each variable can be computed by multiplying together all of the incoming messages from all the other nodes

$$
b_{i}\left(x_{i}\right)=\psi_{i}\left(x_{i}\right) \prod_{k \neq i} m_{k \rightarrow i}\left(x_{i}\right), \quad x_{i} \in \mathcal{A} .
$$

A variant of the sum-product algorithm is the max-product algorithm in which the summation in Eq. (11) is replaced by a maximization over all the symbols in $\mathcal{A}$. In a loopfree MRF graph the sum-product algorithm always converges to the exact marginal probabilities (which corresponds in the case of MIMO detection to a soft decision probability of each symbol $p\left(x_{i} \mid y\right)$ ). In a loop-free MRF graph the max-product variant of the BP algorithm always converges to the most likely configuration [43] (which corresponds to ML decoding in our case). For loop-free graphs, BP is essentially a distributed variant of dynamic programming. The BP message update equations only involve passing messages between neighboring nodes. Computationally, it is thus straightforward to apply the same local message updates in graphs with cycles. In most such models, however, this loopy BP algorithm will not 
compute exact marginal distributions; hence, there is almost no theoretical justification for applying the BP algorithm (one exception is that, for Gaussian graphs, if BP converges, then the means are correct [44]). However, the BP algorithm applied to loopy graphs has been found to have outstanding empirical success in many applications, e.g., decoding LDPC codes [45] and multiuser detection of sparsely spread CDMA [46] where the channel matrix is sparse. The performance of BP in this application may be attributed to the sparsity of the graphs. The cycles in the graph are long, hence the graph has tree-like properties, so that messages are approximately independent and inference may be performed as though the graph was loop-free. The BP algorithm has also been used successfully in image processing and computer vision (e.g., [47]) where the image is represented by a grid-structured MRF that is based on local connections between neighboring nodes.

However, when the graph is not sparse, and is not based on local grid connections, loopy BP almost always fails to converge. Unlike the sparse graphs of LDPC codes, or grid graphs in computer vision applications, the MRF graphs of MIMO channels are completely connected graphs and therefore the associated detection performance is poor. This has prevented the BP from being an asset for the MIMO problem. Fig. 2 shows an example of a BPSK MIMO system based on an $8 \times 8$ matrix and $\mathcal{A}=\{-1,1\}$ (see Section IV for a detailed description of the simulation set-up). As can be seen in Fig. 2, the BP decoder based on the MRF representation (9) has very poor results. Standard techniques to stabilize the BP iterations such as damping the message updates [48] do not help here. Even applying more advanced versions of BP (e.g., Generalized BP and Expectation Propagation [49]) to inference problems on complete MRF graphs yields poor results. The problem here is not in the optimization method but in the cost function that needs to be modified to yield a good approximate solution (see e.g., [50] [51] [52] [53]). In those methods the channel matrix is first converted into a bidiagonal [51], a poly-diagonal [50] or a tree graph [53] forms and, utilizing the effective channel structure, a BP algorithm (which is in these cases simply the Viterbi or the forwardbackward algorithms), is used for detection. These approaches are similar to ours in the sense that in both cases the true channel matrix is replaced by an approximated model that is more suitable for applying BP. The difference is in the approximation that is used. In the other approachs the channel is approximating by a subset of $2 \mathrm{D}$ projections that forms a Markovian process while in our approach we construct an approximation using all the $2 \mathrm{D}$ projections. The MRF in our approach still contains loops but the graphical model is sparse which makes the model suitable for a successful application of the (loopy) belief propagation algorithm.

Shental et al. [21] analyzed the case where the matrix $\mathbf{H}$ is relatively sparse (and has a grid structure) (see Fig. 3). They showed that even under this restricted assumption the BP still does not perform well. As an alternative method they proposed the generalized belief propagation (GBP) algorithm that does work well on the sparse matrix if the algorithm regions are carefully chosen. There are situations where the sparsity assumption makes sense (e.g., 2D intersymbol interference (ISI)

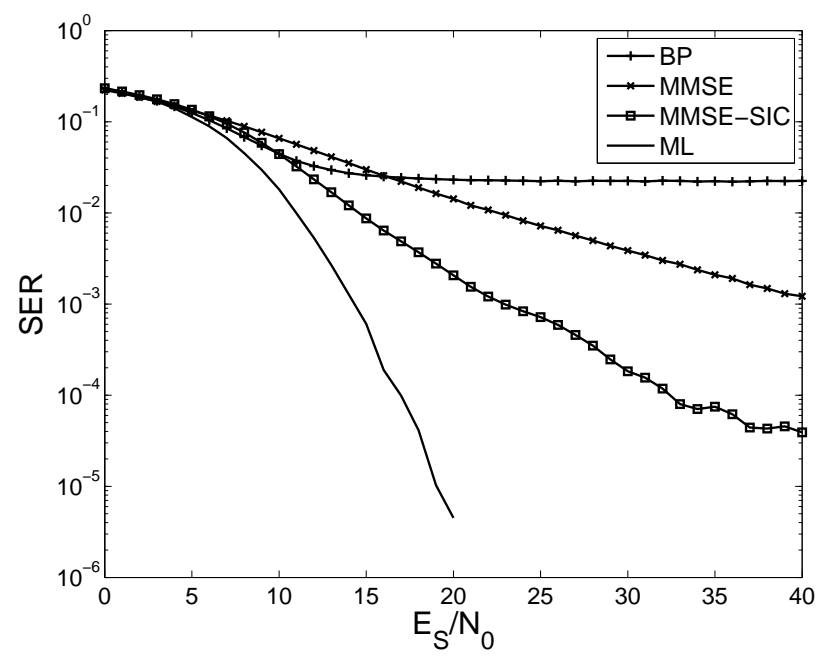

Fig. 2. Decoding results for $8 \times 8$ BPSK real valued system, $\mathcal{A}=\{-1,1\}$.

channels). However, in the MIMO channel model we assume that the channel matrix elements are i.i.d. and Gaussian; hence we cannot assume that the channel matrix $\mathbf{H}$ is sparse.

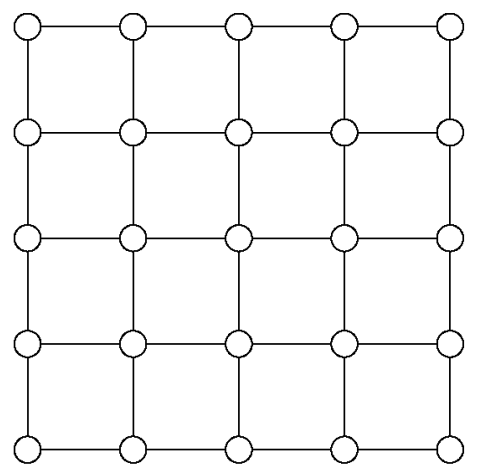

Fig. 3. The MRF grid model corresponds to $5 \times 52 \mathrm{D}$ intersymbol interference (ISI) channels.

In recent years there have also been several attempts to apply BP for densely connected Gaussian graphs. For example consider the MIMO detection problem (8) without the finiteset constraint on the unknown variable $x$ (see e.g., [54]). The Gaussian MRF model, however, is much easier. In this case the complexity of finding the exact solution is a lowdegree polynomial. The BP algorithm can be utilized just to further reduce the complexity. Low-complexity detectors for joint iterative detection and decoding based on factor-graph and message-passing interpretations are detailed in [55] [56].

We note that so far we have assumed a uniform distribution over the transmitted vector $x$. In the case there is a soft input information it can easily be added to the graphical representations described above as a product of a single 
variable potential:

$$
p(x=a)=\prod_{i} p\left(x_{i}=a_{i}\right), \quad a \in \mathcal{A}^{n} .
$$

To simplify presentation the soft-input information is omitted below.

\section{TOMOgRAPHic Decoding of Constrained Linear SYSTEMS}

In this section we present a novel polynomial time algorithm for solving the bounded integer least squares problem. The algorithm outperforms other reduced complexity algorithms with lower complexity. The algorithm has two important steps. The first step is translating the problem into a set of two-dimensional problems. The second step is comprised of solving the two-dimensional problems iteratively by using data received from other two-dimensional problems. This is very similar to tomographic imaging, where an object is reconstructed from its projections on lower-dimensional subspaces. Hence we dub it the Tomographic Least Squares Decoder (TLSD). The difference is that our object is discrete, and the data that are shared among the projections consist of probability distributions.

Our approach is based on an approximation of the probability function:

$$
p\left(x_{1}, . ., x_{n} \mid y\right) \propto \exp \left(-\frac{1}{2 \sigma^{2}}\|\mathbf{H} x-y\|^{2}\right), \quad x \in \mathcal{A}^{n}
$$

which enables a meaningful MRF representation and a successful implementation of the Belief Propagation paradigm. It can be easily verified that:

$$
p(x \mid y) \propto \exp \left(-\frac{1}{2}(x-z)^{\top} \mathbf{C}^{-1}(x-z)\right), \quad x \in \mathcal{A}^{n}
$$

where $z=\left(\mathbf{H}^{\top} \mathbf{H}\right)^{-1} \mathbf{H}^{\top} y$ is the least-squares estimator (5) and $\mathbf{C}=\sigma^{2}\left(\mathbf{H}^{\top} \mathbf{H}\right)^{-1}$ is its variance. Ignoring the finite-set constraint on $x$ we obtain a Gaussian distribution:

$$
f_{z f}(x) \propto \exp \left(-\frac{1}{2}(x-z)^{\top} \mathbf{C}^{-1}(x-z)\right), \quad x \in \mathcal{R}^{n}
$$

Now, instead of marginalizing the true distribution $p(x \mid y)$ we can approximate it by computing the Gaussian marginal of the multivariate Gaussian density $f_{z f}(x)$

$$
p(x \mid y) \propto f_{z f}(x) \approx \prod_{i} f_{z f}\left(x_{i}\right)
$$

and hence, for each $j=1, \ldots, n, p\left(x_{j} \mid y\right) \approx f_{z f}\left(x_{j}\right)$, where

$$
f_{z f}\left(x_{j}\right) \propto \exp \left(-\frac{\left(x_{j}-z_{j}\right)^{2}}{2 c_{j j}}\right)
$$

is the $x_{j}$ marginal Gaussian distribution of $f_{z f}(x)$ and $c_{j j}$ it the $j j$ entry of the matrix C. From the approximation (16) we can extract soft decision results:

$$
p\left(x_{j}=a\right) \propto \exp \left(-\frac{\left(a-z_{j}\right)^{2}}{2 c_{j j}}\right), \quad a \in \mathcal{A} .
$$

Taking the most likely symbol we obtain the ZF approximate solution (6) $\hat{x}_{j}=\arg \min \left|z_{j}-a\right|$.
Motivated by the 1D marginalization (16), we suggest the following 2D marginalization:

$$
p(x \mid y) \propto f_{z f}(x) \approx \prod_{i<j} \phi_{i j}\left(x_{i}, x_{j}\right)
$$

such that $\phi_{i j}\left(x_{i}, x_{j}\right)$ is the 2D marginal Gaussian distribution of $f_{z f}(x)$, i.e., $\phi_{i j}\left(x_{i}, x_{j}\right)$ is

$$
c \cdot \exp \left(-\frac{1}{2}\left(x_{i}-z_{i}, x_{j}-z_{j}\right) \mathbf{C}_{i j}^{-1}\left(x_{i}-z_{i}, x_{j}-z_{j}\right)^{\top}\right)
$$

and $\mathbf{C}_{i j}$ is the $2 \times 2 i j$ sub-matrix of the covariance matrix $\mathbf{C}=\sigma^{2}\left(\mathbf{H}^{\top} \mathbf{H}\right)^{-1}$.

Our approximation approach is, therefore, based on replacing the true distribution $p(x \mid y)$ (14) with the following approximation:

$$
p_{\text {appr }}(x) \propto \prod_{i<j} \phi_{i j}\left(x_{i}, x_{j}\right), \quad x \in \mathcal{A}^{n} .
$$

A natural question is how good this approximation is. Fig. 4 shows that applying ML decoding based on the proposed approximation (20) yields results that are significantly better than the MMSE-SIC algorithm. The probability function $p_{a p p r}(x)$ is factorized in a straightforward way into the two-variable factors $\phi_{i j}\left(x_{i}, x_{j}\right)$. Note that unlike the MRF factorization of the true distribution (9) which is derived from linear-algebra manipulations, the factors of $p_{a p p r}(x)$ have a concrete probabilistic interpretation. The factor $\phi_{i j}\left(x_{i}, x_{j}\right)$ can be viewed as a soft-decision distribution of all the $|\mathcal{A}|^{2}$ possible values of $x_{i}$ and $x_{j}$. Computing the ML solution of the approximated model is, however, still NP hard. Alternatively, we can apply the BP algorithm on the MRF (20). The message from $x_{j}$ to $x_{i}$ is

$$
m_{j \rightarrow i}\left(x_{i}\right)=\sum_{x_{j} \in \mathcal{A}}\left(\phi_{i j}\left(x_{i}, x_{j}\right) \prod_{k \neq i, j} m_{k \rightarrow j}\left(x_{j}\right)\right), \quad x_{i} \in \mathcal{A} .
$$

We show next that the BP algorithm can be effectively utilized for this approximated model although the MRF representation of $p_{\text {appr }}(x)$ (20) still induces a fully connected graph. We show that the MRF $p_{a p p r}(x)$ can be viewed as a representation of a very sparse linear system. Therefore, applying BP algorithm on the MRF (20) is equivalent to utilizing BP on a factor graph model associated with a very sparse linear system.

\section{Geometrical Sparse System Interpretation}

Let $\mathbf{h}_{1}, \ldots, \mathbf{h}_{n}$ be the columns of $\mathbf{H}$ and for each $1 \leq i<$ $j \leq n$ let $\mathbf{A}_{i j}$ be the $m \times(n-2)$ matrix obtained from $\mathbf{H}$ by removing both $i$-th and $j$-th columns. Let

$$
\mathbf{P}_{i j}=\mathbf{I}-\mathbf{A}_{i j}\left(\mathbf{A}_{i j}^{\top} \mathbf{A}_{i j}\right)^{-1} \mathbf{A}_{i j}^{\top}
$$

be the orthogonal projection into the complement of the subspace spanned by $\left\{\mathbf{h}_{k} \mid k \neq i, j\right\}$. Hence

$$
\mathbf{P}_{i j} \mathbf{H} x=\mathbf{P}_{i j} \sum_{k} \mathbf{h}_{k} x_{k}=\mathbf{P}_{i j} \mathbf{h}_{i} x_{i}+\mathbf{P}_{i j} \mathbf{h}_{j} x_{j} .
$$




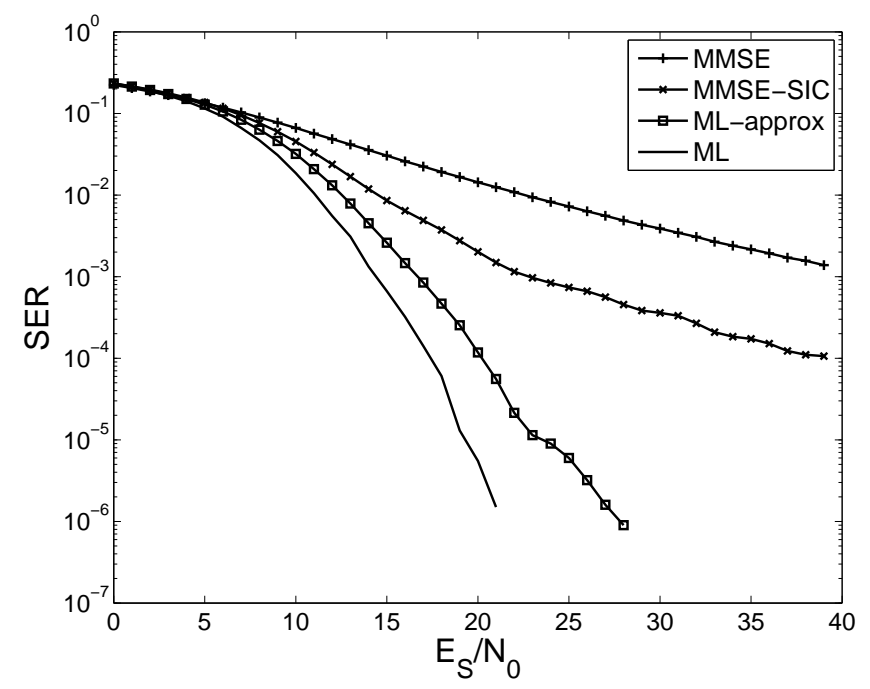

Fig. 4. MIMO detection results for a $8 \times 8$ system, $\mathcal{A}=\{-1,1\}$.

Applying the linear transformation $\mathbf{P}_{i j}$ on both sides of the equation $\mathbf{H} x+\epsilon=y$, yields a set of $m$ equations that depends only on the two variables $x_{i}$ and $x_{j}$ :

$$
\mathbf{P}_{i j} \mathbf{H}_{i j}\left[x_{i}, x_{j}\right]^{\top}+\mathbf{P}_{i j} \epsilon=\mathbf{P}_{i j} y
$$

where $\mathbf{H}_{i j}=\left[\mathbf{h}_{i}, \mathbf{h}_{j}\right]$ and $\mathbf{P}_{i j} \epsilon \sim \mathcal{N}\left(0, \sigma^{2} \mathbf{P}_{i j}\right)$ (note that every orthogonal projection $\mathbf{P}$ satisfies $\left.\mathbf{P}=\mathbf{P}^{2}=\mathbf{P}^{\top}\right)$. The Gaussian density function of $\mathbf{P}_{i j} y$ is:

$f_{i j}\left(\mathbf{P}_{i j} y ; x_{i}, x_{j}\right)=c \cdot \exp \left(-\frac{1}{2 \sigma^{2}}\left\|\mathbf{P}_{i j}\left(y-\mathbf{H}_{i j}\left[x_{i}, x_{j}\right]^{\top}\right)\right\|^{2}\right)$.

Next consider the set of $\left(\begin{array}{l}n \\ 2\end{array}\right)$ linear sparse sub-systems:

$$
\mathbf{P}_{i j} \mathbf{H}_{i j}\left[x_{i}, x_{j}\right]^{\top}+\mathbf{P}_{i j} \epsilon=\mathbf{P}_{i j} y, \quad 1 \leq i<j \leq n
$$

and we further assume that the noise term $\mathbf{P}_{i j} \epsilon \sim$ $\mathcal{N}\left(0, \sigma^{2} \mathbf{P}_{i j}\right)$ is independently sampled for each sub-system. Hence, given the sparse linear system (25), the posterior probability of a vector $x$ (assuming a uniform prior) is:

$$
p_{\text {appr }}^{\prime}(x) \propto \prod_{i<j} \phi_{i j}^{\prime}\left(x_{i}, x_{j}\right), \quad x \in \mathcal{A}^{n}
$$

such that $\phi_{i j}^{\prime}\left(x_{i}, x_{j}\right)=f_{i j}\left(\mathbf{P}_{i j} y ; x_{i}, x_{j}\right)$ viewed as a function of $x_{i}, x_{j}$.

We show next that $p_{a p p r}^{\prime}(x)$ coincides with $p_{a p p r}(x)$ (20) by showing that each factor $\phi_{i j}^{\prime}$ in $p_{a p p r}^{\prime}(x)$ coincides with its corresponding factor $\phi_{i j}$ in $p_{a p p r}(x)$.

Lemma: $\phi_{i j}\left(x_{i}, x_{j}\right)=\phi_{i j}^{\prime}\left(x_{i}, x_{j}\right)$.

Proof: Denote the weighted least-squares solution of the linear system (23) by:

$$
z_{i j}=\left(\mathbf{H}_{i j}^{\top} \mathbf{P}_{i j} \mathbf{H}_{i j}\right)^{-1} \mathbf{H}_{i j}^{\top} \mathbf{P}_{i j} y
$$

It can be easily verified that

$$
\begin{gathered}
\phi^{\prime}\left(x_{i}, x_{j}\right)=f_{i j}\left(\mathbf{P}_{i j} y ; x_{i}, x_{j}\right) \\
=\exp \left(-\frac{1}{2}\left(z_{i j}-E\left(z_{i j}\right)\right)^{\top} \operatorname{Var}\left(z_{i j}\right)^{-1}\left(z_{i j}-E\left(z_{i j}\right)\right)\right) .
\end{gathered}
$$

We show next that $z_{i j}$ is the $i j$ coordinates of the least-square solution $z=\left(\mathbf{H}^{\top} \mathbf{H}\right)^{-1} \mathbf{H}^{\top} y$. To simplify the presentation we rearrange $\mathbf{H}$ such that, without loss of generality, $\mathbf{h}_{i}$ and $\mathbf{h}_{j}$ are the first two columns of $\mathbf{H}$, i.e., $\mathbf{H}=\left[\mathbf{H}_{i j}, \mathbf{A}_{i j}\right]$. Applying the blockwise matrix inversion formula on:

$$
z=\left(\mathbf{H}^{\top} \mathbf{H}\right)^{-1} \mathbf{H}^{\top} y=\left[\begin{array}{ll}
\mathbf{H}_{i j}^{\top} \mathbf{H}_{i j} & \mathbf{A}_{i j}^{\top} \mathbf{H}_{i j} \\
\mathbf{H}_{i j}^{\top} \mathbf{A}_{i j} & \mathbf{A}_{i j}^{\top} \mathbf{A}_{i j}
\end{array}\right]^{-1}\left[\begin{array}{c}
\mathbf{H}_{i j}^{\top} y \\
\mathbf{A}_{i j}^{\top} y
\end{array}\right]
$$

we obtain:

$$
\begin{aligned}
z & =\left[\begin{array}{c}
\left(z_{i}, z_{j}\right)^{\top} \\
\cdots
\end{array}\right] \\
& =\left[\begin{array}{cc}
Q & -Q \mathbf{H}_{i j}^{\top} \mathbf{A}_{i j}\left(\mathbf{A}_{i j}^{\top} \mathbf{A}_{i j}\right)^{-1} \\
\cdots & \cdots
\end{array}\right]\left[\begin{array}{c}
\mathbf{H}_{i j}^{\top} y \\
\mathbf{A}_{i j}^{\top} y
\end{array}\right] \\
& =\left[\begin{array}{c}
\left(\mathbf{H}_{i j}^{\top} \mathbf{P}_{i j} \mathbf{H}_{i j}\right)^{-1} \mathbf{H}_{i j}^{\top} \mathbf{P}_{i j} y \\
\cdots
\end{array}\right]=\left[\begin{array}{c}
z_{i j} \\
\cdots
\end{array}\right] .
\end{aligned}
$$

such that $Q=\left(\mathbf{H}_{i j}^{\top} \mathbf{P}_{i j} \mathbf{H}_{i j}\right)^{-1}$. Hence $z_{i j}=\left(z_{i}, z_{j}\right)^{\top}$ and therefore $E z_{i j}=\left(x_{i}, x_{j}\right)^{\top}$ and $\operatorname{Var}\left(z_{i j}\right)=\mathbf{C}_{i j}$ where $\mathbf{C}_{i j}$ is the $2 \times 2 i j$ sub-matrix of the covariance matrix $\mathbf{C}=\sigma^{2}\left(\mathbf{H}^{\top} \mathbf{H}\right)^{-1}$. Thus we obtain

$$
\begin{gathered}
\phi_{i j}\left(x_{i}, x_{j}\right)=\phi_{i j}^{\prime}\left(x_{i}, x_{j}\right) \\
=c \cdot \exp \left(-\frac{1}{2}\left(x_{i}-z_{i}, x_{j}-z_{j}\right) \mathbf{C}_{i j}^{-1}\left(x_{i}-z_{i}, x_{j}-z_{j}\right)^{\top}\right) .
\end{gathered}
$$

The lemma reveals that $\phi_{i j}\left(x_{i}, x_{j}\right)$ is the density of $\mathbf{P}_{i j} y$ based on the sparse linear system:

$$
\mathbf{P}_{i j} \mathbf{H}_{i j}\left[x_{i}, x_{j}\right]^{\top}+\mathbf{P}_{i j} \epsilon=\mathbf{P}_{i j} y
$$

viewed as a function of $x_{i}$ and $x_{j}$. Therefore, the ML solution of $p_{\text {appr }}(x)$ (20) coincides with the ML solution of a set of $\left(\begin{array}{l}n \\ 2\end{array}\right)$ independently sampled linear sparse sub-systems:

$$
\mathbf{P}_{i j} \mathbf{H}_{i j}\left[x_{i}, x_{j}\right]^{\top}+\mathbf{P}_{i j} \epsilon=\mathbf{P}_{i j} y, \quad i<j .
$$

In other words:

$$
\arg \max _{x} p_{\text {appr }}(x)=\arg \min _{x} \sum_{i<j}\left\|\mathbf{P}_{i j}\left(y-\mathbf{H}_{i j}\left[x_{i}, x_{j}\right]^{\top}\right)\right\|^{2} .
$$

The two models are equal and yield the same MRF representation. The geometrical model provides an interpretation of the probabilistic model presented in the previous section as a model of a sparse set of linear equations. Of course, the BP algorithms applied on the two models are the same.

The crux of our argument is that by applying the 2D projections we shift from the original likelihood function $p(x \mid y)$ (8) to a very similar function $p_{a p p r}(x)$ that is much easier to optimize. The sparsity of the system (32) makes $p_{a p p r}(x)$ a much smoother function than the original likelihood function since changing the value of one of the variables influences just a small number of equations in the linear system (32). The smoothness of $p_{\text {appr }}(x)$ makes it possible to apply an effective iterative search. A similar situation occurs in LDPC codes [45] where the sparsity of the parity-check equations results in a smooth likelihood function. We dub the BP algorithm based on the 2D Gaussian marginalization (20) the "BP2D algorithm". 


\section{The BP2D/TLSD Algorithm:}

Input: A constrained linear LS problem: $\mathbf{H} x+\epsilon=y$ a noise level $\sigma^{2}$ and a finite symbol set $\mathcal{A}$.

Goal: Find (approx. to) $\arg \min _{x \in \mathcal{A}^{n}}\|\mathbf{H} x-y\|^{2}$.

Preprocessing:

Compute $z=\left(\mathbf{H}^{\top} \mathbf{H}\right)^{-1} \mathbf{H}^{\top} y$ and $\mathbf{C}=\sigma^{2}\left(\mathbf{H}^{\top} \mathbf{H}\right)^{-1}$.

For each pair $1 \leq i<j \leq n$

For every $a, a^{\prime} \in \mathcal{A}$, compute:

$\phi_{i j}\left(a, a^{\prime}\right)=\exp \left(-\frac{1}{2}\left(z_{i}-a, z_{j}-a^{\prime}\right) \mathbf{C}_{i j}^{-1}\left(z_{i}-a, z_{j}-a^{\prime}\right)^{\top}\right)$

s.t. $\mathbf{C}_{i j}$ is the $2 \times 2 i j$ sub-matrix of the matrix $C$. End

Max-Product iteration:

$$
\begin{aligned}
& m_{j \rightarrow i}(a)=\max _{a^{\prime} \in \mathcal{A}}\left(\phi_{i j}\left(a, a^{\prime}\right) \prod_{k \neq i, j} m_{k \rightarrow j}\left(a^{\prime}\right)\right) \\
& \hat{x}_{i}=\arg \max _{a \in \mathcal{A}} \prod_{j \neq i} m_{j \rightarrow i}(a)
\end{aligned}
$$

Sum-Product iteration:

$$
\begin{aligned}
& m_{j \rightarrow i}(a)=\sum_{a^{\prime} \in \mathcal{A}}\left(\phi_{i j}\left(a, a^{\prime}\right) \prod_{k \neq i, j} m_{k \rightarrow j}\left(a^{\prime}\right)\right) \\
& \operatorname{belief}_{i}(a) \propto \prod_{j \neq i} m_{j \rightarrow i}(a)
\end{aligned}
$$

TLSD Iteration:

$$
\text { E-step: } \begin{aligned}
w_{i j}(a) \propto \theta_{i}(a) \sum_{a^{\prime} \in \mathcal{A}} \theta_{j}\left(a^{\prime}\right) \phi_{i j}\left(a, a^{\prime}\right), \\
i=1, \ldots, n, \quad a \in \mathcal{A}
\end{aligned}
$$

M-step: $\hat{\theta}_{i}(a)=\frac{1}{n-1} \sum_{j \neq i} w_{i j}(a)$

Fig. 5. The BP2D/TLSD Algorithm.

There are a few implementation issues regarding the proposed BP2D algorithm that require discussion. Even when using the proposed smooth approximate cost function there is no guarantee that loopy BP always converges. To overcome this problem we can compute the hard-decision solution at the end of each BP iteration and after the BP converges, or after a pre-defined number of iterations, we can choose the solution that minimizes the exact cost function $\|\mathbf{H} x-y\|^{2}$. Otherwise, we can use variants of BP that always converge (e.g., [57]).

To implement the Belief Propagation algorithm, either the sum-product algorithm or the max-product algorithm must be chosen. The sum-product algorithm computes the marginal distributions of each node, while the max-product algorithm computes the MAP estimate of the whole MRF. We choose to use the max-product algorithm for the following reasons. First, the detection performance of the two variants of the BP algorithm are very similar. However, as we show in the experiment section, it takes the max-product fewer iterations to converge than it takes for the sum-product.

The map-product avoids complicated exponent-log computations and it can be carried out in the log domain just by using addition operations, which is useful for practical implementations. The sum-product messages can be viewed as a soft max version of the max-product. The level of softness is a function of the noise level parameter $\sigma^{2}$. In contrast, the max-product algorithm does not depend on $\sigma^{2}$ and can be applied in cases where the noise level is unknown or even when it is not constant but changes between transmitted frames.

Another implementation issue is the complexity of the algorithm and the message update schedule. The complexity of a straightforward implementation of the BP algorithm is $O\left(n^{3}|\mathcal{A}|\right)$ where $n$ is the number of unknown variables (number of transmit antennas). The complexity is $n^{3}$ because at each iteration we need to compute a message between each two variables and computing a single message is linear in $n$. The following efficient implementation (based on a certain scheduling of message passing) can reduce the BP complexity to a quadratic function of the number of transmit antennas:

$$
\begin{aligned}
& \text { for } j=1, \ldots, n \\
& \qquad \begin{array}{l}
\operatorname{sum}_{j}\left(x_{j}\right)=\sum_{k \neq j} m_{k \rightarrow j}\left(x_{j}\right) \\
\text { for } i=1, \ldots, n \\
\quad m_{j \rightarrow i}\left(x_{i}\right)=\max _{x_{j} \in \mathcal{A}}\left(\log \phi_{i j}\left(x_{i}, x_{j}\right)+\right. \\
\left.\qquad \operatorname{sum}_{j}\left(x_{j}\right)-m_{i \rightarrow j}\left(x_{j}\right)\right)
\end{array}
\end{aligned}
$$

We show in the next section that the BP2D performs better than the MMSE and even better than its sequential version MMSE-SIC. We have found that we can improve the BP2D algorithm by computing both the BP2D solution and the MMSE-SIC solution and choose the one with the higher likelihood. The resulting combined detector works much better than its two components. The experimental results for BP2D presented in the next section are based on this combined detector.

\section{AN EM VARIANT OF THE ITERATIVE ALGORITHM}

In the previous section we transformed the original linear system $y=\mathbf{H} x+\epsilon$ into a new sparse linear system

$$
\mathbf{P}_{i j} \mathbf{H}_{i j}\left[x_{i}, x_{j}\right]^{\top}+\mathbf{P}_{i j} \epsilon=\mathbf{P}_{i j} y, \quad 1 \leq i<j \leq n
$$

where each equation depends on just two variables. Recalling Eq. (20), the likelihood function of $x$ based on the new sparse system (where the noise terms of the sub-systems are assumed to be independent) is:

$$
p_{\text {appr }}(x) \propto \prod_{i<j} \phi_{i j}\left(x_{i}, x_{j}\right), \quad x \in \mathcal{A}^{n}
$$

and we want to find the most-likely solution: $\hat{x}=$ $\arg \max p_{\text {appr }}(x)$. This maximization problem for the sparse system is still NP hard. As an alternative to the BP approach described above we present here an approximate solution based on the well known EM algorithm. There are two reasons why we use the EM here. First we empirically validated that the EM based algorithm performance on the MIMO examples we tested is similar to the performance of the BP algorithm. Second, the general EM theory guarantees a monotone convergence of the iterative algorithm (unlike loopy belief propagation).

To apply the EM algorithm for finding $\max p_{a p p r}(x)$, we first define a new two-step generative probabilistic model that 
is a relaxation of the model defined by (20). The model parameters are as follows. For each variable $x_{i}$ we associate a discrete distribution $\theta_{i}=\left\{\theta_{i}(a) \mid a \in \mathcal{A}\right\}$ on the $|\mathcal{A}|$ discrete symbols. The observed random variables is the set $\left\{\mathbf{P}_{i j} y \mid i<j\right\}$. For each pair $i<j$, the observed vector $\mathbf{P}_{i j} y$ is independently generated in two steps:

- Two hidden random variables are sampled. We sample $x_{i}$ according to the multinomial distribution $\theta_{i}$, i.e., $p\left(x_{i}=\right.$ $a)=\theta_{i}(a)$ and similarly we sample $x_{j} \sim \theta_{j}$.

- An additive Gaussian noise is sampled $\epsilon_{i j} \sim N\left(0, \sigma^{2}\right)$ and we compute $\mathbf{H}_{i j}\left[x_{i}, x_{j}\right]^{\top}+\mathbf{P}_{i j} \epsilon_{i j}$. The observed vector $\mathbf{P}_{i j} y$ is viewed as the result of this computation.

The density function of $\mathbf{P}_{i j} y$ according to this probabilistic generative model, denoted by $g_{i j}\left(\mathbf{P}_{i j} y ; \theta_{i}, \theta_{j}\right)$, is:

$$
\begin{gathered}
g_{i j}\left(\mathbf{P}_{i j} y ; \theta_{i}, \theta_{j}\right)=\sum_{a, a^{\prime} \in \mathcal{A}} p\left(x_{i}=a ; \theta_{i}\right) p\left(x_{j}=a^{\prime} ; \theta_{j}\right) \times \\
\times f\left(\mathbf{P}_{i j} y \mid x_{i}=a, x_{j}=a^{\prime}\right) \\
=\sum_{a, a^{\prime} \in \mathcal{A}} \theta_{i}(a) \theta_{j}\left(a^{\prime}\right) \phi_{i j}\left(a, a^{\prime}\right)
\end{gathered}
$$

such that

$$
\begin{gathered}
f\left(\mathbf{P}_{i j} y \mid x_{i}=a, x_{j}=a^{\prime}\right)= \\
c \cdot \exp \left(-\frac{1}{2 \sigma^{2}}\left\|\mathbf{P}_{i j}\left(y-\mathbf{h}_{i} a-\mathbf{h}_{j} a^{\prime}\right)\right\|^{2}\right)=\phi_{i j}\left(a, a^{\prime}\right) .
\end{gathered}
$$

The likelihood of a parameter set $\theta$ based on this hiddenvariable model is:

$$
\begin{aligned}
& g(y ; \theta)=\prod_{i<j} g_{i j}\left(\mathbf{P}_{i j} y ; \theta_{i}, \theta_{j}\right) \\
= & \prod_{i<j} \sum_{a, a^{\prime} \in \mathcal{A}} \theta_{i}(a) \theta_{j}\left(a^{\prime}\right) \phi_{i j}\left(a, a^{\prime}\right) .
\end{aligned}
$$

In the model (20), $x$ is a hidden random variable that is sampled once for all the sub-systems. In the two step model defined above $\left\{x_{i}, x_{j}\right\}$ are hidden random variables that can have different values in different sub-systems. Note that if the distribution parameters $\left\{\theta_{i}\right\}$ are all restricted to deterministic probability vectors (delta functions) then the continuous likelihood function (37) is reduced to the discrete likelihood function (20). For each $x=\left\{x_{1}, \ldots, x_{n}\right\} \in \mathcal{A}^{n}$ we can associate a parameter set $\theta^{x}$ as follows:

$$
\theta_{i}^{x}(a)=1_{\left\{x_{i}=a\right\}}, \quad i=1, \ldots, n \quad a \in \mathcal{A}
$$

It can easily be verified from Eq. (20) and (37) that in this case $p_{a p p r}(x)=g\left(y ; \theta^{x}\right)$. In other words, finding the ML estimation for $\theta$ over the deterministic probability vectors is exactly the problem of finding the ML solution for the sparse linear system (20). Hence the probabilistic model we defined extends the original likelihood function from $\mathcal{A}^{n}$ into a continuous space $\Omega^{n}$ such that $\Omega=\left\{\theta: \mathcal{A} \rightarrow[0,1] \mid \sum_{a} \theta(a)=1\right\}$ which can be viewed as the convex-hull of $\mathcal{A}$. The continuous model that is based on hidden variables is suitable for applying the EM algorithm. The model we defined is based on latent random variables that are associated with each sparse equationset.
We next derive the EM algorithm for the model described above. The log-likelihood function of the complete data is:

$$
\begin{aligned}
& \log f(x, y ; \theta)=\sum_{i<j} \sum_{a \in \mathcal{A}} \sum_{a^{\prime} \in \mathcal{A}} 1_{\left\{x_{i}=a\right\}} 1_{\left\{x_{j}=a^{\prime}\right\}} \times \\
& \quad \times\left(\log \theta_{i}(a)+\log \theta_{j}\left(a^{\prime}\right)+\log f\left(\mathbf{P}_{i j} y \mid x_{i}=a, x_{j}=a^{\prime}\right)\right)
\end{aligned}
$$

The hidden variables are all the pairs $\left\{\left(x_{i}, x_{j}\right) \mid i<j\right\}$ such that each pair is independently sampled for the corresponding linear sub-system. The observed data is the random variable set $\left\{\mathbf{P}_{i j} y \mid i<j\right\}$. The EM auxiliary function, therefore, is:

$$
\begin{gathered}
Q\left(\theta, \theta^{0}\right)=E\left(\log f(x, y ; \theta) \mid y ; \theta^{0}\right) \\
=\sum_{i<j} \sum_{a \in \mathcal{A}} \sum_{a^{\prime} \in \mathcal{A}} p\left(x_{i}=a, x_{j}=a^{\prime} \mid \mathbf{P}_{i j} y ; \theta^{0}\right) \times \\
\times\left(\log \theta_{i}(a)+\log \theta_{j}\left(a^{\prime}\right)\right)+\mathrm{const}
\end{gathered}
$$

such that $\theta^{0}$ is the current estimate of the unknown parameter set. Utilizing Bayes rule we obtain:

$$
p\left(x_{i}=a \mid \mathbf{P}_{i j} y ; \theta^{0}\right)=\frac{\theta_{i}^{0}(a) \sum_{a^{\prime} \in \mathcal{A}} \theta_{j}^{0}\left(a^{\prime}\right) \phi_{i j}\left(a, a^{\prime}\right)}{g_{i j}\left(\mathbf{P}_{i j} y ; \theta_{i}^{0}, \theta_{j}^{0}\right)}
$$

such that $g_{i j}$ is defined in (35). This concludes the E-step. The updated parameter set in the M-step is derived through maximization of the auxiliary function, .i.e.,

$$
\hat{\theta}=\arg \max _{\theta} Q\left(\theta, \theta^{0}\right)=\arg \max _{\theta} Q\left(\theta_{1}, \ldots, \theta_{n}, \theta^{0}\right) .
$$

It is easily verified from (40) that this maximization can be done for each $\theta_{i}$ independently. Maximizing the expression $\sum_{a}\left(\sum_{j \neq i} p\left(x_{i}=a \mid \mathbf{P}_{i j} y ; \theta^{0}\right)\right) \log \theta_{i}(a)$, given the constraint $\sum_{a} \theta_{i}(a)=1$, finally yields the EM parameter updating formula:

$$
\hat{\theta}_{i}(a)=\frac{1}{n-1} \sum_{j \neq i} p\left(x_{i}=a \mid \mathbf{P}_{i j} y ; \theta\right), \quad i=1, \ldots, n, \quad a \in \mathcal{A} .
$$

The EM algorithm requires appropriate initialization, as it can get stuck on local maxima of the likelihood function. The ZF solution (or the MMSE) can be used for initialization:

$$
\theta_{i}(a) \propto \exp \left(-\frac{\left(z_{i}-a\right)^{2}}{2 c_{i i}}\right), \quad a \in \mathcal{A} .
$$

The BP2D/TLSD algorithm is summarized in Fig. 5.

\section{EXPERIMENTAL RESULTS}

In this section we provide simulation results for the proposed detector over various MIMO systems. We assume a frame length of 100 , i.e., the channel matrix $\mathbf{H}$ is constant for 100 channel uses. The channel matrix comprised iid elements drawn from a zero-mean normal distribution of unit variance. We used 10,000 realizations of the channel matrix. This resulted in $10^{6}$ vector messages. The performance of the proposed algorithm is shown as a function of the variance of the additive noise $\sigma^{2}$. The signal-to-noise ratio (SNR) is 


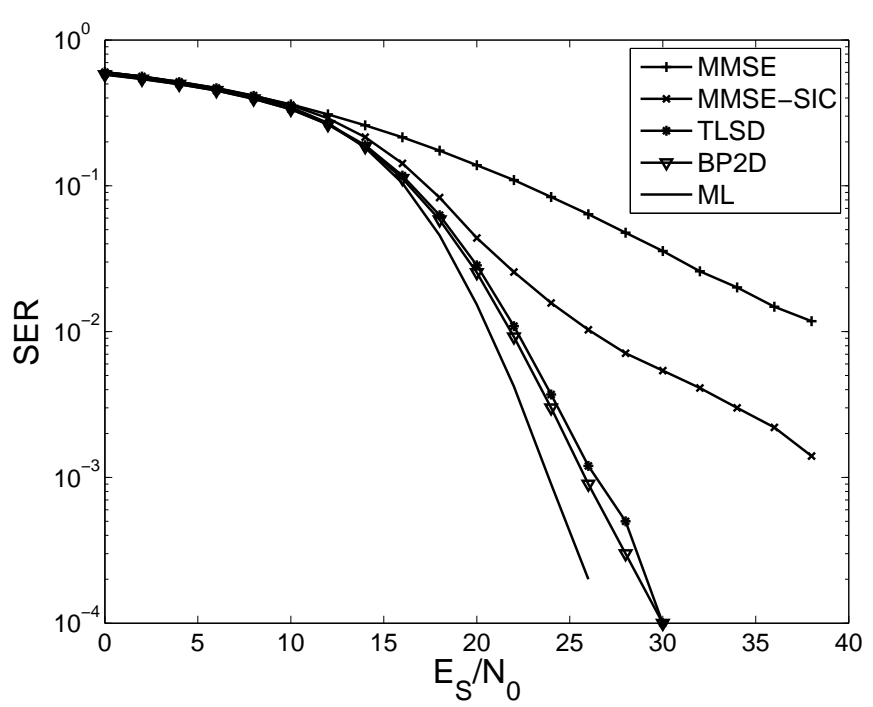

Fig. 6. Results for a $6 \times 6$ system, $\mathcal{A}=\{ \pm 1, \pm 3\}$.

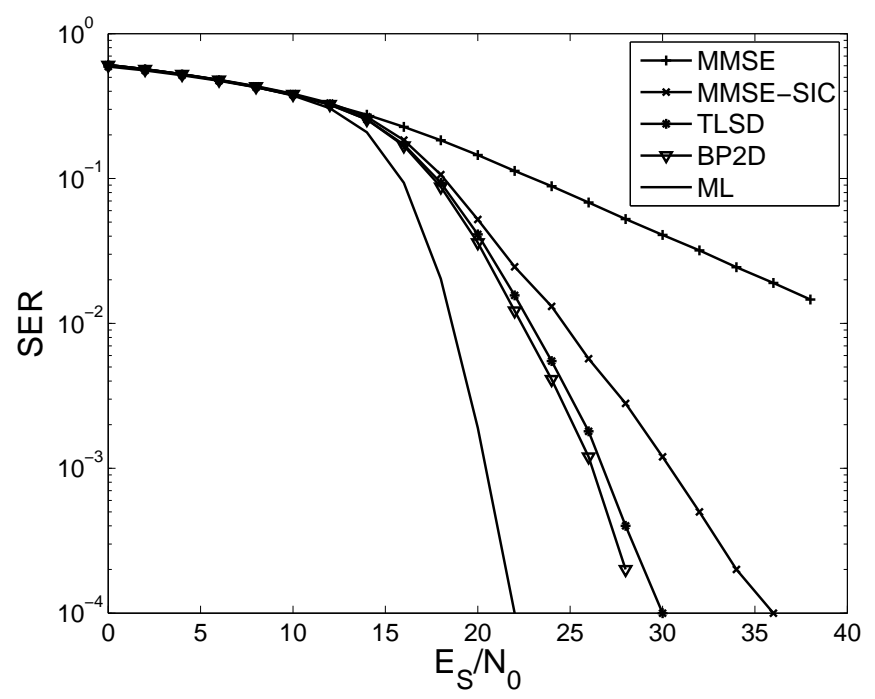

Fig. 7. Results for a $16 \times 16$ system, $\mathcal{A}=\{ \pm 1, \pm 3\}$.

defined as $10 \log _{10}\left(E_{s} / N_{0}\right)$ where $E_{s} / N_{0}=\frac{n e}{\sigma^{2}}$ ( $n$ is the number of variables, $e=\frac{1}{|\mathcal{A}|} \sum_{a \in \mathcal{A}} a^{2}$ and $\sigma^{2}$ is the variance of the Gaussian additive noise). In this paper we present two variants of iterative algorithms, namely BP2D and TLSD. The two algorithms are based on the same 2D approximation and yield very similar results. The EM based algorithm has a theoretical guarantee of convergence. In practice the EM algorithm is more conservative and it requires more iterations until convergence.

Fig. 6 shows the symbol error rate (SER) versus SNR for a $6 \times 6, \mathcal{A}=\{ \pm 1, \pm 3\}$ MIMO system. The performance of the proposed $2 \mathrm{D}$ projection methods BP2D and TLSD are compared to ML detection and to other linear suboptimal algorithms: the linear MMSE and the sequential MMSE. In all our experiments the number of BP2D iterations was limited to 10 and the number of the TLSD iterations was limited to 20. It can be seen that the 2D-projection based algorithms are significantly better than the MMSE-SIC at the same computational complexity. Fig. 7 shows similar results for a $16 \times 16 \mathcal{A}=\{ \pm 1, \pm 3\}$ system and Fig. 8 shows similar results for a $6 \times 6$ complex MIMO system with $|\mathcal{A}|=64$ and 9 shows similar results for a $6 \times 6$ complex MIMO system with $|\mathcal{A}|=64$.

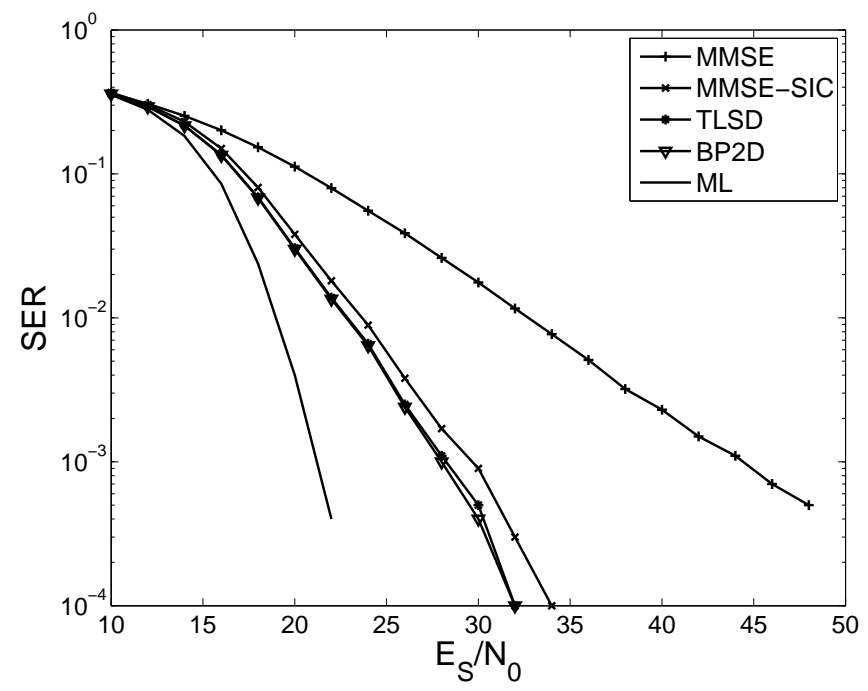

Fig. 8. Results for a $6 \times 6$ complex system, $|\mathcal{A}|=16$.

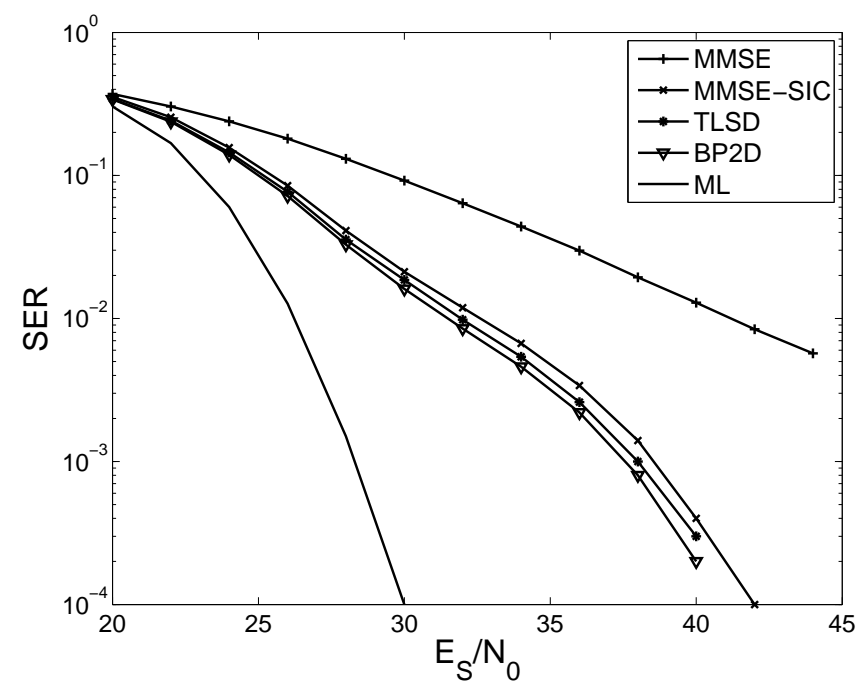

Fig. 9. Results for a $6 \times 6$ complex system, $|\mathcal{A}|=64$.

\section{DISCUSSION}

While the proposed methods provide excellent performance, it is worth noting that we obtain a-posteriori probabilities for each variable that can be used to improve performance. This is done by applying a Schnorr-Euchner sphere decoding algorithm [9], where we order the symbols according to their a-posteriori probabilities given by our ILS. This can lead to very close to optimal performance with significantly reduced complexity. This is because by computing the a-posteriori probabilities, we have a much higher probability of finding the true solution during the first search, therefore significantly 
reducing the search radius. Solving integer least squares problems is an important problem in many fields. We have proposed a novel technique based on the tomographic principle of reconstruction from projections. We showed that the method always converges. Furthermore, the proposed method has good performance competitive to all other polynomial algorithms for solving the problem as demonstrated in simulations. Finally, we have outlined how the method can be extended to provide a-posteriori probabilities per bit for use in coded communication systems.

\section{REFERENCES}

[1] S.M. Kay, Fundumentals of statistical signal processing: Estimation theory, PTR, Prentice Hall, 1993.

[2] P.J.G. Teunissen, "Success probability of integer GPS ambiguity rounding and bootstrapping," Journal of Geodesy, vol. 72, no. 10, pp. 606-612, 1998.

[3] Bert M. Kampes, Radar interferometry, Springer, 2006.

[4] E. Agrell, T. Eriksson, A. Vardy, and K. Zeger, "Closest point search in lattices," IEEE Trans. Inf. Theory, vol. 48, no. 8, pp. 2201-2214, 2002.

[5] J. C. Lagarias and A. M. Odlyzko, "Solving low-density subset sum problems," J. ACM, vol. 32, no. 1, pp. 229-246, 1985.

[6] P. van Emde Boas, "Another NP-complete partition problem and the complexity of computing short vectors in a lattice," Tech. Rep. Dept. of Mathematics, Univ. of Amsterdam 81-04, 1981.

[7] G.J. Foschini, "Layered space-time architecture for wireless communication in a fading environment when using multi-element antennas," Bell Labs Technical Journal, vol. 1, no. 2, pp. 41-59, 1996.

[8] M. Pohst, "On the computation of lattice vectors of minimal length, successive minima and reduced bases with applications," SIGSAM Bull., vol. 15 , no. 1, pp. 37-44, 1981.

[9] C.P. Schnorr and M. Euchner, "Lattice basis reduction: Improved practical algorithms and solving subset sum problems," Journal of Mathematical Programming, vol. 66, no. 1-3, pp. 181-199, Aug. 1994.

[10] J. Jalden and B. Ottersten, "On the complexity of sphere decoding in digital communications," IEEE Trans. Signal Processing, vol. 53, no. 4, pp. 1474-1484, 2005.

[11] E.G. Larsson and J. Jalden, "Fixed-complexity soft mimo detection via partial marginalization," IEEE Transactions on Signal Processing, vol. 56, no. 8, pp. $3397-3407,2008$.

[12] A. Wiesel, Y.C. Eldar, and S.S. Shitz, "Semidefinite relaxation for detection of 16-QAM signaling in MIMO channels," IEEE Signal Processing Letters, vol. 12, no. 9, pp. 653-656, Sept. 2005.

[13] N.D. Sidiropoulos and Z.-Q. Luo, "A semidefinite relaxation approach to MIMO detection for high-order QAM constellations," IEEE Signal Processing Letters, vol. 13, no. 9, pp. 525-528, Sept. 2006.

[14] P. H. Tan and L. K. Rasmussen, "The application of semidefinite programming for detection in CDMA," IEEE Journal on Selected Areas in Communications, vol. 19, no. 8, pp. 1442-1449, 2001.

[15] A. B. Reid, A. J. Grant, and A. P. Kind, "Low complexity list detection for high-rate multiple-antenna channels," Proceedings. Int. Symposium on Information Theory, 2003.

[16] A.P. Kind and A.J. Grant, "On estimating the symbol APP in MIMO systems," Proceedings. Int. Symposium on Information Theory, 2004.

[17] A.P. Kind and A.J. Grant, "Very-high rate space-time coding," Information Theory Workshop, pp. 341-344, 2003.

[18] A.P. Kind and A.J. Grant, "A tree search method for iterative decoding of underdetermined multiuser systems," Proceedings. Int. Symposium on Information Theory, pp. 189-193, 2005.

[19] L. K. Rasmussen, T. J. Lim, and T.M. Aulin, "Breadth-first maximum likelihood detection in multiuser CDMA," IEEE Trans. on Communications, vol. 45, no. 10, pp. 1176-1178, 1997.

[20] P. H. Tan, L. K. Rasmussen, and T. J. Lim, "Constrained maximumlikelihood detection in CDMA," vol. 49, no. 1, pp. 142-153, 2001.

[21] O. Shental, N. Shental, S. Shamai (Shitz), I. Kanter, A.J. Weiss, and Y. Weiss, "Discrete-input two-dimensional Gaussian channels with memory: Estimation and information rates via graphical models and statistical mechanics," IEEE Trans. Inf. Theory, vol. 54, no. 4, pp. 1500 1513,2008

[22] A. Leshem and J. Goldberger, "MIMO decoding based on stochastic reconstruction from multiple projections," IEEE Int. Conf. on Acoustics, Speech and Signal Processing (ICASSP), 2009.
[23] M. Grotschel, L. Lovasz, and A. Schriver, "Geometric Algorithms and Combinatorial Optimization," Springer Verlag, 2nd edition, 1993.

[24] M. Ajtai, "The shortest vector problem in L2 is NP-hard for randomized reductions," Proc. Annual ACM Symposium on Theory of Computing, pp. 10-19, 1998.

[25] B. Hassibi, "An efficient square-root algorithm for BLAST," Proc. IEEE Intl. Conf. Acoustic, Speech, Signal Processing, pp. 5-9, 2000.

[26] C. Studer, A. Burg, and H. Bolcskei, "Soft-output sphere decoding: algorithms and vlsi implementation," IEEE Journal on Selected Areas in Communications, vol. 26, no. 2, pp. 290 -300, 2008.

[27] G. D. Golden, G. J. Foschini, R. A. Valenzuela, and P. W. Wolniansky, "Detection algorithm and initial laboratory results using V-BLAST space-time communication architecture," Electron. Letters, vol. 35, no. 1, pp. 14-16, 1999.

[28] U. Fincke and M. Pohst, "Improved methods for calculating vectors of short length in a lattice, including a complexity analysis," Mathematics of Computation, vol. 44, no. 170 , pp. 463-471, 1985.

[29] C.P. Schnorr and M. Euchner, "Lattice basis reduction: Improved practical algorithms and solving subset sum problems," Journal of Mathematical Programming, vol. 66, no. 1-3, pp. 181-199, 1994.

[30] J. Boutros, N. Gresset, L. Brunel, and M. Fossorier, "Soft-input softoutput lattice sphere decoder for linear channels," Proc. IEEE Global Communications Conference, Dec. 2003.

[31] C. Studer, A. Burg, and H. Bölcskei, "Soft-output sphere decoding: Algorithms and VLSI implementation," IEEE Journal on Selected Areas in Communications, vol. 26, no. 2, pp. 290-300, 2008.

[32] J. Jalden and B. Ottersten, "On the complexity of sphere decoding in digital communications," IEEE Trans. Signal Processing, vol. 53, no. 4, pp. 1474-1484, 2005.

[33] C. B. Schlegel, Z.-J. Xiang, and S. Roy, "Projection receiver: a new efficient multi-user detector," Global Telecommunications Conference, GLOBECOM, pp. 142-146, 1995.

[34] C. B. Schlegel, S. Roy, P. D. Alexander, and Z.-J. Xiang, "Multiuser projection receivers," IEEE Journal on Selected Areas in Communications, vol. 14, no. 8, pp. 1610-1618, 1996.

[35] P. D. Alexander, L. K. Rasmussen, and C. B. Schlegel, "A linear receiver for coded multiuser CDMA," IEEE Trans. on Communications, vol. 45, no. 5, pp. 605-610, 1997

[36] C. B. Schlegel and A. Grant, Coordinated Multi-User Communications, Springer Publishers, 2006.

[37] L. K. Rasmussen, T. J. Lim, and P. D. Alexander, "A new geometrical interpretation of the decorrelator in multiuser CDMA," Global Telecommunications Conference, GLOBECOM, vol. 3, pp. 1561-1565, 1996.

[38] D. Pham, K.R. Pattipati, P.K. Willett, and J. Luo, "A generalized probabilistic data association detector for multiple antenna systems," IEEE Communications Letters, vol. 8, no. 4, pp. 205-207, 2004.

[39] Y. Jia, C.M. Vithanage, C. Andrieu, and R.J. Piechocki, "Probabilistic data association for symbol detection in mimo systems," Electronics Letters, vol. 42, no. 1, pp. 38-40, 2006.

[40] G. Latsoudas and N.D. Sidiropoulos, "A hybrid probabilistic data association-sphere decoding detector for multiple-input-multiple-output systems," Signal Processing Letters, IEEE, vol. 12, no. 4, pp. 309-312, 2005.

[41] J. S. Yedidia, W. T. Freeman, and Y. Weiss, "Understanding belief propagation and its generalizations," Proc. Int. Joint Conf. on Artificial Intelligence, Aug. 2001.

[42] E. Sudderth and W. Freeman, "Signal and image processing with belief propagation," IEEE Signal Processing Magazine, DSP Applications Column, vol. 25, no. 2, pp. 114-141, 2008.

[43] J. Pearl, Probabilistic Reasoning in Intelligent Systems, San Mateo CA: Morgan Kaufman, 1988.

[44] Y. Weiss and W.T. Freeman, "Correctness of belief propagation in Gaussian graphical models of arbitrary topology," Neural Computation, vol. 13, no. 10, pp. 2173-2200, 2001.

[45] R. G. Gallager, Low density parity check codes, M.I.T. Press, 1963.

[46] D. Guo and C. Wang, "Multiuser detection of sparsely spread CDMA," IEEE Journal on Selected Areas in Communications, vol. 26, no. 3, pp. 421-431, 2008

[47] P. F. Felzenszwalb and D. P. Huttenlocher, "Efficient belief propagation for early vision," Int. Journal of Computer Vision, vol. 70, no. 1, pp. 41-54, 2006.

[48] K. Murphy, Y. Weiss, and M. Jordan, "Loopy belief propagation for approximate inference: An empirical study," Proc. Uncertainty in Artificial Intelligence, pp. 467-475, July 1999.

[49] T. Minka and Y. Qi, "Tree-structured approximations by expectation propagation," Proc. Neural Information Processing Systems, Dec. 2004. 
[50] S. Yoon and S. Lee, "A detection algorithm for multi-input multi-output (MIMO) transmission using poly-diagonalization and trellis decoding," IEEE Journal on Selected Areas in Communications, vol. 26, no. 8, pp. 993-1002, 2008.

[51] W. Jiang, Y. Li, and X. Yu, "Truncation for low-complexity MIMO signal detection," IEEE Trans. on Inform. Theory, vol. 53, pp. 1564$1571,2007$.

[52] J. Goldberger and A. Leshem, "Pseudo prior belief propagation for densely connected discrete graphs," Proc. IEEE Information Theory Workshop, Jan. 2010.

[53] J. Goldberger and A. Leshem, "MIMO detection for high-order QAM based on a Gaussian tree approximation," IEEE Trans. Information Theory, vol. 57, pp. 4973-4982, 2011.

[54] D. Bickson, O. Shental, P. H. Siegel, J. K. Wolf, and D. Dolev, "A Gaussian belief propagation based multiuser detection," Proc. IEEE Int. Symp. Information Theory, July 2008.

[55] J. Boutros and G. Caire, "Iterative multiuser joint decoding: unified framework and asymptotic analysis," IEEE Trans. on Information Theory, vol. 48, no. 7, pp. 1772-1793, 2002.

[56] G. Caire, R. R. Muller, and T. Tanaka, "Iterative multiuser joint decoding: optimal power allocation and low-complexity implementation," IEEE Trans. on Information Theory, vol. 50, no. 9, pp. 1950-1973, 2004.

[57] A. Globerson and T. Jaakkola, "Fixing max-product: convergent message passing algorithms for MAP LP-relaxations," Proc. Neural Information Processing Systems, 2008.

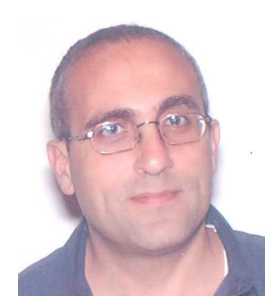

Jacob Goldberger received the B.Sc. degree (cum laude) in mathematics and computer science from the Bar-Ilan University, Ramat Gan, Israel, the M.Sc. degree (cum laude) in mathematics, and the Ph.D. degree in Engineering, from the Tel-Aviv University, Tel-Aviv, Israel. In 2004 he joined the School of Electrical and Computer Engineering, Bar-Ilan University, Ramat Gan, Israel, where he is currently a faculty member. His main research interests include machine learning with applications to information theory, computer vision, medical imaging, speech recognition and natural language processing.

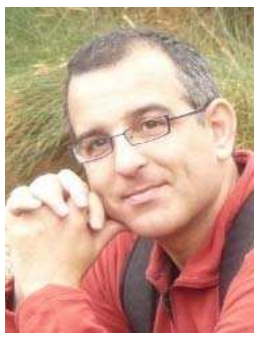

Amir Leshem (M'98, SM'06) received the B.Sc.(cum laude) in mathematics and physics, the M.Sc. (cum laude) in mathematics, and the Ph.D. in mathematics all from the Hebrew University, Jerusalem, Israel, in 1986,1990 and 1998 respectively. From 1998 to 2000 he was with Faculty of Information Technology and Systems, Delft university of technology, The Netherlands, as a postdoctoral fellow working on algorithms for the reduction of terrestrial electromagnetic interference in radioastronomical radio-telescope antenna arrays and signal processing for communication. From 2000 to 2003 he was director of advanced technologies with Metalink Broadband where he was responsible for research and development of new DSL and wireless MIMO modem technologies and served as a member of ITU-T SG15, ETSI TM06, NIPPNAI, IEEE 802.3 and 802.11. From 2000 to 2002 he was also a visiting researcher at Delft University of Technology. He is one of the founders of the new school of electrical and computer engineering at Bar-Ilan university where he is currently an Associate Professor and head of the Signal Processing track. From 2003 to 2005 he also was the technical manager of the U-BROAD consortium developing technologies to provide $100 \mathrm{Mbps}$ and beyond over copper lines. His main research interests include multichannel wireless and wireline communication, applications of game theory to dynamic and adaptive spectrum management of communication networks, array and statistical signal processing with applications to multiple element sensor arrays and networks, wireless communications, radio-astronomical imaging and brain research, set theory, logic and foundations of mathematics. 